\title{
Ionization and Transmission Efficiency in an Electrospray Ionization- Mass Spectrometry Interface
}

\author{
Jason S. Page, Ryan T. Kelly, Keqi Tang, and Richard D. Smith \\ Biological Sciences Division, Pacific Northwest National Laboratory, Richland, Washington, USA
}

\begin{abstract}
The ionization and transmission efficiencies of an electrospray ionization (ESI) interface were investigated to advance the understanding of how these factors affect mass spectrometry (MS) sensitivity. In addition, the effects of the ES emitter distance to the inlet, solution flow rate, and inlet temperature were characterized. Quantitative measurements of ES current loss throughout the ESI interface were accomplished by electrically isolating the front surface of the interface from the inner wall of the heated inlet capillary, enabling losses on the two surfaces to be distinguished. In addition, the ES current lost to the front surface of the ESI interface was spatially profiled with a linear array of 340- $\mu \mathrm{m}$-diameter electrodes placed adjacent to the inlet capillary entrance. Current transmitted as gas-phase ions was differentiated from charged droplets and solvent clusters by measuring sensitivity with a single quadrupole mass spectrometer. The study revealed a large sampling efficiency into the inlet capillary ( $>90 \%$ at an emitter distance of $1 \mathrm{~mm}$ ), a global rather than a local gas dynamic effect on the shape of the ES plume resulting from the gas flow conductance limit of the inlet capillary, a large $(>80 \%)$ loss of analyte ions after transmission through the inlet arising from incomplete desolvation at a solution flow rate of $1.0 \mu \mathrm{L} / \mathrm{min}$, and a decrease in analyte ions peak intensity at lower temperatures, despite a large increase in ES current transmission efficiency. (J Am Soc Mass Spectrom 2007, 18, 1582-1590) (c) 2007 American Society for Mass Spectrometry
\end{abstract}

$\mathrm{E}$ lectrospray ionization (ESI) has become a prominent ionization technique for a broad range of chemical and biological applications of mass spectrometry (MS) [1,2] because of its ability to create intact, multiply charged gas-phase ions (from, e.g., biomolecules in solution) and its facile coupling with on-line separation techniques [such as liquid chromatography (LC)] [3-6]. The sensitivity of ESI-MS is largely determined by the effectiveness of producing gas-phase ions from analyte molecules in solution (ionization efficiency) and the ability to transfer the charged species from atmospheric pressure to the low-pressure region of the mass analyzer (transmission efficiency) [7-10].

Ionization efficiency is affected by a number of factors, such as flow rate, interface design, solvent composition, and analyte properties. In general, ionization efficiency increases as the liquid flow to the ES emitter decreases [11-13]. The primary reason for this increase is the production of smaller charged droplets at the lower flow rates [11, 14]. The smaller droplets enable more efficient solvent evaporation and fewer coulombic fission events are required to create gasphase ions [11, 14]. Also, the ES current in cone-jet mode increases approximately as the square root of the

Address reprint requests to Richard D. Smith, Pacific Northwest National Laboratory, Biological Systems Analysis and Mass Spectrometry, 3335 Q Ave., P.O. Box 999, MS K8-98, Richland, WA 99352, USA. E-mail: rds@pnl.gov volumetric flow rate [14], increasing the number of available charges per analyte molecule as the flow rate decreases. Finally, smaller initial droplets and increased amount of charge available per analyte molecule improve the ionization of analytes with lower surface activity, improving quantitation and reducing matrix suppression effects [15, 16]. In addition to its importance for transmission efficiency, the ESI interface on the mass spectrometer also plays a key role in ionization efficiency [17]. Adding energy to the charged droplets-such as using heated nitrogen as a background gas-enhances desolvation and liberates more analyte ions [17-20].

Ion transmission efficiency, also defined as the fraction of ES current that enters the mass analyzer, has traditionally been limited by losses at the mass spectrometer inlet and at the skimmer [7, 21]. It has been estimated that only about one out of every $10^{3}-10^{5}$ analyte ions generated by ESI at atmospheric pressure is actually detected using present instrument designs [7, $10,22]$. Analyte loss at the inlet arises in part from a rapidly expanding charged droplet/ion plume caused by coulombic repulsion and the shape of the electric field, which disperses the sample across an area much larger than the inlet to the mass spectrometer. A larger sampling orifice or inlet capillary with a larger inner diameter (id) can improve transmission efficiency $[8,23$, 24]; however, improvements are limited by the practical constraints on the larger pumps needed to overcome 
the increase of the gas load into the instrument and maintain the desired pressure in the first vacuum stage. In addition, the gas flow in the inlet can change from laminar to turbulent at larger id values, and cause further ion loss.

Analyte loss at the skimmer occurs because only a portion of the ions entrained in the expanding jet are sampled. The small size of the skimmer aperture (typically $1 \mathrm{~mm}$ in diameter) limits gas throughput, enabling differential pumping, but can also result in large ES current losses. The electrodynamic ion funnel was developed to replace the skimmer and reduce the ES current loss in this region [25-29]. The ion funnel is an adaptation of a stacked ring ion guide [30] where the exit of the ion guide tapers down to the diameter of the conductance-limiting orifice. In contrast to the skimmer interface, the ion funnel can focus ions over a broad $\mathrm{m} / \mathrm{z}$ range exiting the inlet capillary and transmit them with minimal loss.

Limitations in both the ionization and transmission efficiencies lead directly to reduced ESI-MS sensitivity. Improving ESI-MS sensitivity can often enable new sample-limited analyses, such as micro-dissected cells, micro-biopsies, or even single cells. It would be beneficial to better understand and characterize the underlying mechanisms of ESI ionization and transmission efficiency to improve sensitivity. There are several previous studies that have focused on different aspects of the ESI interface, such as the mechanisms of the ES process [7, 31, 32], the profiling of the ES plume [33], and the transmission of the ES current through an orifice or capillary inlet [8,34-36]. Building upon these studies, we have developed a platform with which ion production, loss, and transmission are characterized together using a common ESI interface geometry. The ES currents lost to the front surface of a mass spectrometer were spatially profiled using a linear array of small electrodes placed adjacent to the heated inlet capillary, and the distribution of current losses was measured as the ion plume was sampled into the instrument inlet. The effects of different ES variables on the ion transmission efficiency were obtained by differentiating the ES current hitting the front of the inlet capillary from the current lost inside the capillary, while also detecting the transmitted current. Additionally, analyte MS peak intensity was used to correlate ES current measurements with the analyte ionization/transmission efficiency. Combined, the results provide a better understanding of ESI and transmission efficiency and can be used for improving ESI and other atmospheric-pressure ion source interfaces.

\section{Experimental}

\section{Sample Preparation}

A solvent mixture for ES was prepared by combining purified water (Barnstead Nanopure Infinity System, Dubuque, IA, USA) and methanol (HPLC grade, Fisher
Scientific, Fair Lawn, NJ, USA) in a 1:1 ratio and adding acetic acid (Sigma-Aldrich, St. Louis, MO, USA) at $1 \%$ $\mathrm{v} / \mathrm{v}$. A reserpine (Sigma-Aldrich) stock solution was prepared in a 1:1 solution of $n$-propanol (Fisher Scientific) and purified water and then diluted in the ES solvent to a final concentration of $1 \mu \mathrm{M}$.

\section{ESI Capillary Inlet Electrode Array}

To spatially profile the current in the ES plume at the entrance of the inlet, a linear array of 23 electrodes was incorporated into the front section of the heated capillary assembly. The heated capillary inlet consisted of a 490- $\mu \mathrm{m}$-id stainless steel capillary (Part \# 89935K82, McMaster-Carr, Los Angeles, CA, USA) silver soldered in the center of the stainless steel body. The metal immediately below the inlet was removed and a stainless steel vise was constructed to hold 23 Kapton-coated $340-\mu \mathrm{m}$-outer diameter (od) copper wires in a line, as shown in Figure 1a and b. The front of the inlet was machined flat and polished with 2000 grit sandpaper (Norton Abrasives, Worcester, MA, USA), making the ends of the wires an array of electrically isolated electrodes. The other ends of the wires were connected to an electrical breadboard with two outputs: one to common ground and another to a Keithley picoammeter (Model No. 6485, Keithley, Cleveland, OH, USA) referenced to ground. To record the ES current from one of the electrodes, the corresponding wire was selected and manually moved from the common ground output to the picoammeter input and 100 consecutive measurements were averaged using the built-in data acquisition capabilities of the picoammeter. ES currents were profiled with the inlet connected to a ground potential and coupled to a vacuum chamber operating at 2 Torr, which served as a stand-alone ESI interface to simulate the front end of a mass spectrometer (see below).

\section{Measuring ES Current Throughout the ESI Interface}

Measurements of ES currents having an impact on and transmitted through a heated capillary inlet were made using a separate ESI source and a heated capillary interface that was constructed from a four-way reducing cross with a 6-in. main flange, as shown in Figure 1c. ${ }^{\circ}$ A rough ${ }^{\circ}$ pump ${ }^{\circ}$ (E1M18, ${ }^{\circ}$ BOC ${ }^{\circ}$ Edwards, Wilmington, MA, USA) was used to pump the chamber (2 Torr), and a strain gauge (BOC Edwards) measured the pressure. The front flange served as a holder for the capillary inlet and the back flange contained a BNC electrical feedthrough. The inlet was heated to $120^{\circ} \mathrm{C}$ by two 100-W cartridge heaters (Watlow, St. Louis, MO, USA) and the temperature was regulated by a temperature controller (Model CN9000A, Omega, Stanford, CT, USA). Three separate charge collector electrodes were used to detect the ES current lost to the front of the inlet capillary, inner wall of the capillary, and transmitted 
a)

b)

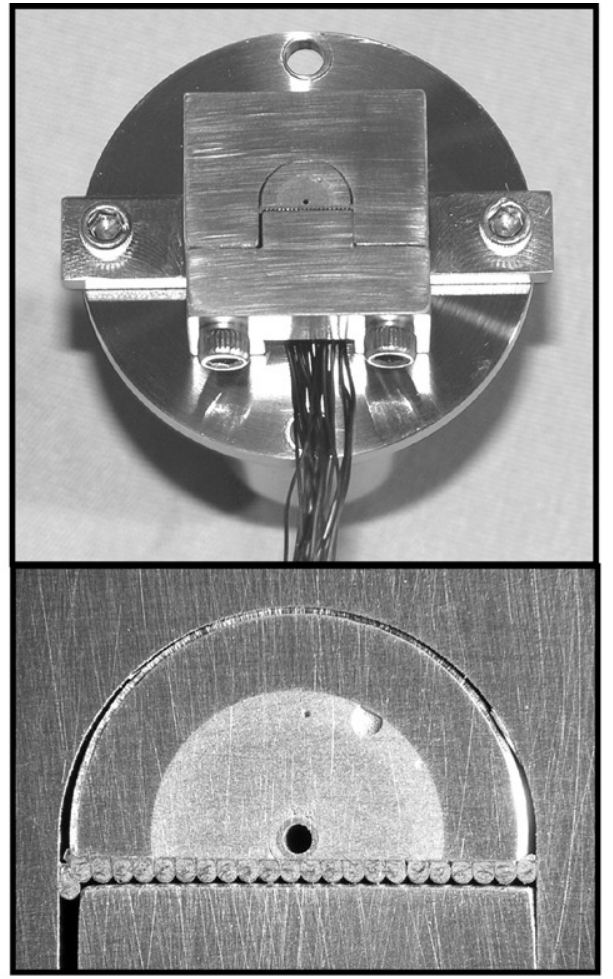

c)

$4 \mathrm{~cm}$ diameter

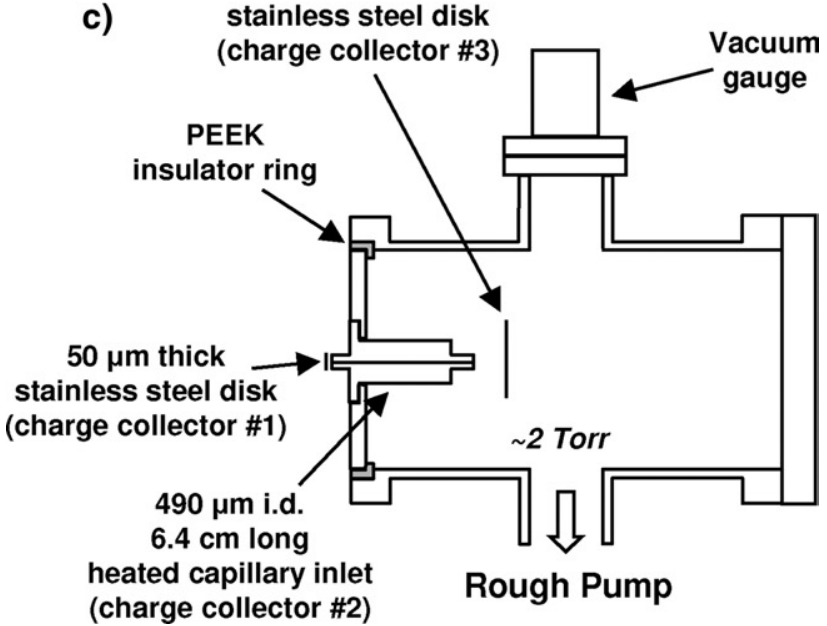

Figure 1. Photographs of the linear electrode array incorporated with a metal capillary inlet and a schematic depiction of the setup used to detect the ES current transmission through the inlet. (a) A view of the entire front of the inlet that displays the electrode array and the metal vise used to compress the ends of wires against the inlet. (b) A close-up view of the array showing the close proximity of the electrodes to the entrance of the inlet capillary. (c) A drawing of the experimental setup used to detect the ES current lost to the front of the interface, the inner wall of the inlet capillary, and the current transmitted through the inlet. The first two charge collectors were made using a thin layer of cyanoacrylate to adhere and electrically isolate a metal disk to the front of the interface. Wires connected the disk and the inlet capillary to a picoammeter separately. A metal plate positioned $\sim 1 \mathrm{~cm}$ from the inlet capillary exit served as the third charge collector.

through the inlet. The first electrode was made by attaching a 50- $\mu \mathrm{m}$-thick, 7.6-mm-diameter stainless steel disk to the front of the heated capillary inlet using a cyanoacrylate adhesive (Loctite Prism 411, Henkel Technologies, Avon, OH, USA). The cyanoacrylate formed a strong bond that was very thin and had a high dielectric strength that electrically isolated the metal disk from the rest of the inlet capillary. A $500-\mu \mathrm{m}$-id hole in the center of the disk was aligned with the $490-\mu \mathrm{m}$-id capillary in the inlet by inserting a 483$\mu \mathrm{m}$-od wire gauge in the inlet and sliding the disk down on the wire gauge during the gluing process. After a few minutes, the wire gauge was removed. A Kapton-coated copper wire was attached to the front of the metal disk using a conductive epoxy (Part \# CW2400, ITW Chemtronics, Kennesaw, GA, USA) and was used to connect the disk to common ground or a picoammeter during current measurements. To measure the ES current hitting the inner wall of the inlet capillary, a metal wire was used to connect the inlet to the common ground or the picoammeter. The ES current transmitting through the inlet capillary was detected by a 4-cm-diameter stainless steel disk positioned $1 \mathrm{~cm}$ behind the exit of the inlet capillary. This disk was connected using a BNC vacuum electrical feedthrough to common ground or a picoammeter.

\section{ESI Setup}

An ES emitter was prepared by chemical etching as described ${ }^{\circ}$ previously ${ }^{\circ}[37]^{\circ}$ from $^{\circ} a^{\circ}$ section $^{\circ}$ of ${ }^{\circ} 77-\mu \mathrm{m}$-id/ $360-\mu \mathrm{m}$-od fused silica capillary tubing (Polymicro Technologies, Phoenix, AZ, USA). The same emitter was used for all the experiments. The emitter was connected to a transfer capillary and a $100-\mu \mathrm{L}$ syringe (Hamilton, Las Vegas, NV, USA) by a stainless steel union, which also served as the connection point for the ES voltage. All solutions were infused from a Harvard Apparatus Model 22 syringe pump (Holliston, MA, USA). Voltages were applied to the ES emitter by a Bertan high-voltage power supply (Model 205B-03R, Hicksville, NY, USA). An x-y-z stage (Newport, Irvine, CA) and a stereo microscope were used to accurately control the placement and movement of the ES emitter.

\section{MS Instrumentation}

An Agilent MSD1100 (Santa Clara, CA, USA) singlequadrupole mass spectrometer equipped with a custom-built ESI/ion funnel source was used; the ion funnel was similar to what was described previously [38]. Mass ${ }^{\circ}$ spectra ${ }^{\circ}$ of ${ }^{\circ} a^{9}$ reserpine ${ }^{\circ}$ solution ${ }^{\circ}$ were ${ }^{\circ}$ acquired with a $100-1500 \mathrm{~m} / \mathrm{z}$ range in positive ESI mode with a $0.1 \mathrm{~m} / \mathrm{z}$ step size. Each spectrum used was the result of a 10-scan average to reduce the effects of any intensity fluctuations in the ES. The same heated capillary inlets used on the stand-alone ESI interface were also used on the single-quadrupole mass spectrometer along with the same ES emitter, syringe, syringe pump, and ES conditions (voltage, distance, flow rate, etc.). 


\section{Results and Discussion}

\section{Distance of the Emitter to the Inlet}

The distance of the ES emitter to the sampling inlet is adjustable on many ESI interfaces. This gap is often between 2 and $10 \mathrm{~mm}$ with a larger gap generally used for higher solution flow rates. At shorter distances, more of the ES plume will be sampled into the instrument; however, a smaller gap reduces the droplet evaporation time, which can in turn lower ionization efficiency. We characterized the ES current loss and transmission in a common ESI interface as a function of the emitter distance to the inlet. The ESI interface incorporated a $6.4-\mathrm{cm}$-long inlet capillary heated to $120^{\circ} \mathrm{C}$. A solvent mixture was supplied at $0.5 \mu \mathrm{L} / \mathrm{min}$. Figure $^{\circ} 2 \mathrm{a}^{\circ}$ plots $^{\circ}$ the $^{\circ} \mathrm{ES}^{\circ}$ current $^{\circ}$ detected $^{\circ}$ on ${ }^{\circ}$ the ${ }^{\circ}$ inlet surface (the front of the instrument), lost to the inner wall of the capillary, and transmitted into the vacuum chamber. Total ES current was obtained by summing the currents from the individual charge collectors. The data are an average from three separate acquisitions, with standard deviations shown with error bars. The ES voltage was increased slightly at the longer emitter to inlet distances to maintain the stable cone-jet mode. At a distance of $1 \mathrm{~mm}$, most of the ES current successfully entered the inlet; only about $7 \%$ of the current hit the front surface. A majority of the current was lost inside the capillary $(\sim 69 \%)$, leaving roughly $24 \%$ transmitted into the vacuum region of the interface. As the emitter distance increased, the amount of ES current lost on the front of the interface quickly increased and began to level off at $5 \mathrm{~mm}$. This increase, as well as the decrease in the spray current sampled into the inlet, is attributed to an expansion of the ES plume. Both the amount of charge hitting the inside capillary wall and the transmitted current also decreased with increasing emitter gap. The effectiveness of this setup to accurately detect the ES current was tested by repeating the experiment with the pump to the ESI interface off (eliminating the inlet gas flow). The result showed no significant change to the total ES current and almost all the current being lost on the front surface of the interface. Only a small amount of current penetrated into the inlet and was detected on the inner capillary wall. Its value decreased from about $4 \mathrm{nA}$ at $1 \mathrm{~mm}$ to $<1 \mathrm{nA}$ as the distance was increased. With the pump off, no current was transmitted through the inlet. This verified that there was good electrical isolation of the front and inside walls of the capillary, and there was also no significant background current that could mar the results.

The amount of ES current lost to the front of the interface as a function of emitter distance is easily understood by relating the ES plume diameter to the area effectively sampled by the gas dynamics of the inlet; however, the current transmission through the inlet capillary is a more complex process, in which both charge repulsion and ion diffusion play key roles. This is ${ }^{\circ}$ illustrated ${ }^{\circ}$ in $^{\circ}$ Figure $^{\circ} 2 \mathrm{~b}^{\circ}$ where ${ }^{\circ}$ the ${ }^{\circ}$ transmission $^{\circ}$ effi- a)

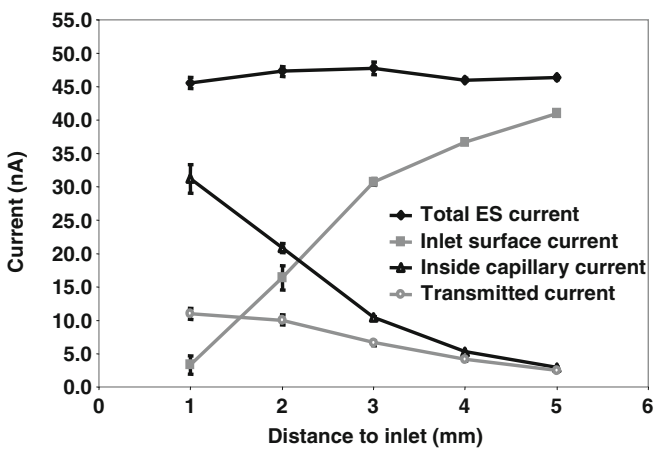

b)

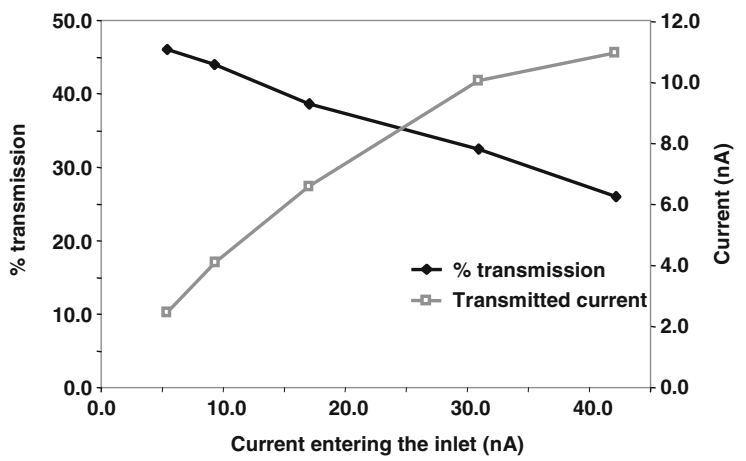

c)

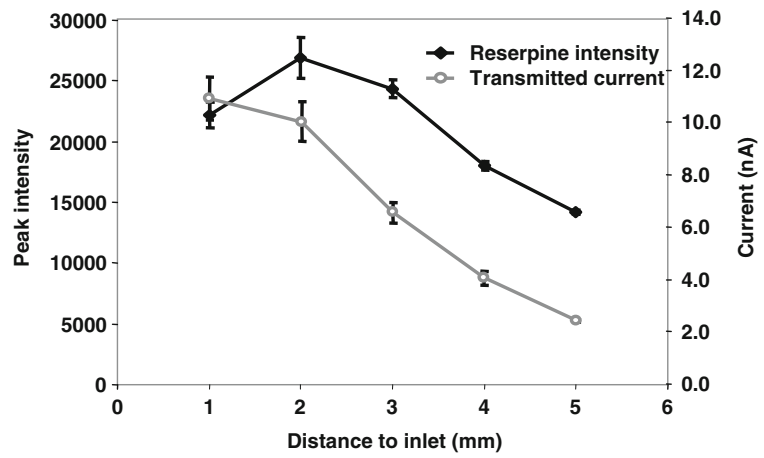

Figure 2. Ionization and transmission efficiency in an ESI interface as a function of the distance of the ES emitter to the inlet and total ES current entering the inlet with a solution flow rate of $0.5 \mu \mathrm{L} / \mathrm{min}$. (a) Plots of the detected ES current on the front of the interface, the inside wall of the capillary, and transmitted through the capillary. The total ES current is a summation of the current detected on the three charge collectors. (b) The transmission efficiency of the capillary inlet and the current transmitted through the inlet as function of the amount of ES current entering the inlet. The amount of current entering the inlet was determined by adding the current detected on the inside walls of the capillary and the current transmitted through the inlet from a. The transmission efficiency (\% transmission) was calculated by dividing the transmitted current by the amount of current entering the inlet capillary and multiplying by 100 . (c) Reserpine peak intensity and transmitted current as a function of ES emitter distance to the inlet.

ciency of the inlet capillary (\% transmission) and the transmitted current are plotted with respect to the amount of current entering the capillary. The current entering the capillary was obtained by summing the transmitted current and the current hitting the inner wall of the capillary for each emitter distance from Figure 2a. The transmission efficiency was calculated by dividing the transmitted current by the current entering 
the capillary. The larger the current sampled by the inlet, the larger the current transmitted through, but with lower transmission efficiency. In other words, the transmission efficiency is not constant (the amount of transmitted current is not proportional to the amount of current entering the capillary) but decreases as the amount of current increases. This is explained, in a large part, by space-charge effects where the ion plume expansion rate increases as the total charge of the ion cloud (occupying the same volume) increases. This is explained ${ }^{\circ}$ in $^{\circ}$ more $^{\circ}$ detail $^{\circ}$ by $^{\circ}$ Lin $^{\circ}$ and ${ }^{\circ}$ Sunner ${ }^{\circ}[36],{ }^{\circ}$ who theorized that an atmospheric-pressure ion interface transitions into a space-charge-dominated ion source at ion currents $>100 \mathrm{pA}$, well below the ion currents in this experiment.

Besides space-charge, diffusion is another factor leading to ES current loss inside the inlet capillary. Although diffusion is unaffected by the number of ions entering the capillary, other factors, such as charged particle size (ion, cluster, or droplet), capillary dimensions, residence time, and temperature have an impact on diffusional ion loss. Both diffusion and space-charge effects are dependent on mobility. There is a large distribution of particle sizes in the ES plume ranging from gas-phase ions to droplets. In addition, desolvation and droplet fission events can continue to occur inside the capillary, causing the mobilities to change as the particles travel through the capillary.

Characterizing the ES current in an ESI interface using a simple charge collector does not provide a complete picture of the efficiency of an ESI interface because analytes trapped in clusters and droplets are indistinguishable from liberated gas-phase ions. To obtain information on the ionization efficiency, the same ESI interface was placed on a single-quadrupole mass spectrometer and the emitter distance experiment was ${ }^{\circ}$ repeated ${ }^{\circ}$ using $^{\circ}$ the ${ }^{\circ}$ same $^{\circ} \mathrm{ES}^{\circ}$ conditions. $^{\circ}{ }^{\circ}$ Figure ${ }^{\circ} 2 \mathrm{c}$ shows the peak intensity of reserpine and the transmit-

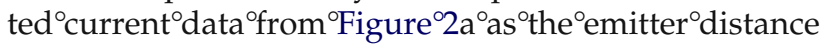
was increased. A key observation from this experiment was that the reserpine peak intensity increases slightly around 2 to $3 \mathrm{~mm}$ and then starts to decrease at greater distances. In contrast, the transmitted current continually decreases as the distance increases. The intensity of the reserpine peak relates directly to the fraction of ES current constituting gas-phase reserpine ions transmitted into the mass analyzer by the ESI interface (as long as the intensity of the ion beam is well within the dynamic range of the instrument). Because the peak increases slightly at 2- and 3-mm distances, there are more gas-phase reserpine ions in the interface than at the 1-mm distance. This may be related to increased desolvation and fission events in the gap between the emitter and inlet as a result of the longer distances. Although the 1-mm distance gave a larger transmitted ES current, less of the reserpine was fully ionized and possibly trapped in droplets and solvent clusters. Additional insight can be gained by analyzing the data from the 4-mm emitter distance. Here, the reserpine intensity is similar to that for the 1-mm distance, indicating that the number of gas-phase reserpine ions transmitted into the mass analyzer by the ESI interface changed very little between the two distances. However, the total transmitted current decreased from 11 to $4 \mathrm{nA}$ as the distance was changed from 1 to $4 \mathrm{~mm}$. The amount of reserpine sampled into the interface should also have a similar increase at the shorter distance (because the majority of the mass transferred from the ES is carried by charged droplets), but the reserpine peak intensity does not significantly change, indicating that almost all the reserpine gained by decreasing the distance is not fully ionized and is either neutral or in association with droplets and clusters. In addition, by using the ratio of the increase in total transmitted ion current moving from a 4 - to $1-\mathrm{mm}$ distance $(\sim 7 \mathrm{nA})$ over the total transmitted at the 1-mm distance $(\sim 11$ $\mathrm{nA}$ ), we can conclude that $>60 \%$ of the reserpine transmitted into the interface at the 1-mm distance does not constitute fully liberated gas-phase ions and therefore does not increase the reserpine peak intensity.

Increasing the distance of the ES emitter to the mass spectrometer inlet improves ionization efficiency at the cost of reducing the transmission of the ES current into the inlet. ${ }^{\circ}$ Based $^{\circ}$ on $^{\circ}$ the ${ }^{\circ}$ data $^{\circ}$ in ${ }^{\circ}$ Figure $^{\circ} 2,{ }^{\circ} a^{\circ} 3-\mathrm{mm}^{\circ}$ distance resulted in roughly $65 \%$ of the ES current being lost before the inlet. To spatially profile this loss, we fabricated a linear array of small round electrodes onto a heated capillary inlet with the same dimensions as the inlet used in $^{\circ}$ the ${ }^{\circ}$ previous $^{\circ}$ transmission $^{\circ}$ study $^{\circ}\left(\right.$ see $^{\circ}$ Figure $\left.^{\circ} 1\right){ }^{\circ}$ This allowed us to probe the current density across a slice of the front surface of the interface while the inlet was attached to a mass spectrometer or the stand-alone ESI interface. Figure 3 a shows ${ }^{9}$ the plots $^{\circ}$ of the detected ${ }^{\circ}$ current from each individual electrode with respect to various emitter distances to the inlet. It is important to note that the data were collected while there was a vacuum applied to the chamber behind the inlet, and thus the inlet was actively sampling the ES plume. The ES plume width for an emitter distance of $2 \mathrm{~mm}$ was about $2.5 \mathrm{~mm}$. This width increases to about $7 \mathrm{~mm}$ at a distance of $5 \mathrm{~mm}$. Data could not be collected for the 1-mm distance because no current was detected on any of the electrodes as a result of the very high sampling efficiency. Also shown in the plot is a drawn circle that indicates the approximate size and location of the inlet. It is remarkable to observe how large the ES plume is compared to the size of the inlet, and it is easy to understand why this is an area where considerable analyte loss can occur.

Another area from which we can gain information by using the linear electrode array is the effect that the gas flow at the mass spectrometer inlet has on the sampling of the ES plume. To accomplish this, current density profiles were obtained at an ES emitter distance of $4 \mathrm{~mm}$ with repeated pump on (with gas sampling) and off. The resulting ES current profiles are plotted and compared in ${ }^{\circ}$ Figure $3 \mathrm{~b}$. These Thata $^{\circ}$ show $^{\circ}$ the ${ }^{\circ}$ entire ${ }^{\circ}$ plume is affected by the gas flow as opposed to a local effect where only the current near the inlet is changed. For 
a)

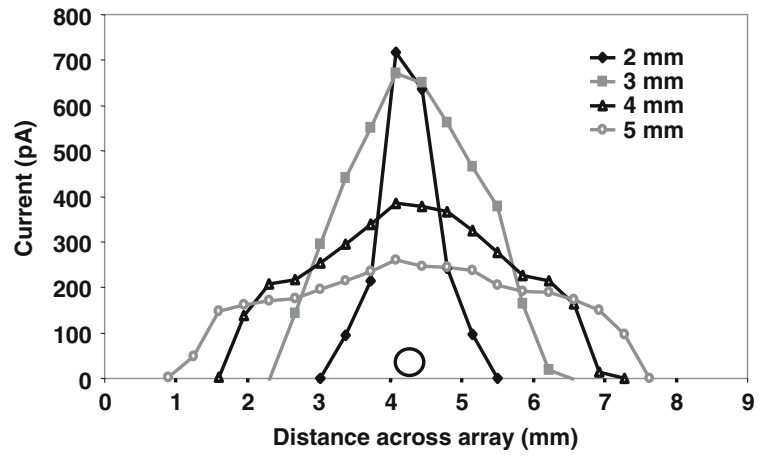

b)

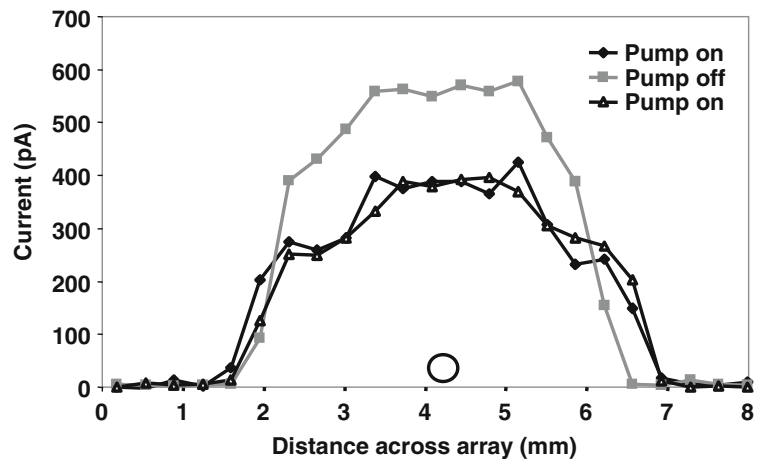

Figure 3. Profiles of the ES current having an impact on the front of the ESI interface. The data points were obtained by detecting the ES current on each electrode in the linear array. The solution flow rate was $0.5 \mu \mathrm{L} / \mathrm{min}$. The drawn circle represents the approximate size and location of the capillary inlet. (a) ES current profiles for different ES emitter distances to the inlet. (b) ES current profiles showing the influence of the gas dynamics of the inlet on the ES plume. Background gas was sampled into the capillary inlet while the pump was on. No gas was sampled into the inlet when the pump was off.

instance, the ES currents at about 2 and $6 \mathrm{~mm}$ are reduced by roughly $35 \%$ even though the electrodes are about $2 \mathrm{~mm}$ away from the inlet. Additionally, the areas under the respective pump "on" and "off" profiles can be used to obtain approximate volumes of current and provide another independent measure of inlet sampling efficiency $(\sim 20 \%)$.

\section{ESI Flow Rate}

It has been well established that operating ESI at low flow $^{\circ}$ rates $^{\circ}$ improves $^{\circ}$ ionization $^{\circ}$ efficiency ${ }^{\circ}[11-13] .{ }^{\circ}$ We further confirmed this by performing transmission efficiency experiments for a distance of $2 \mathrm{~mm}$ between the emitter and the inlet capillary using a solvent mixture or reserpine solution pumped at flow rates between 0.1 and $2.0 \mu \mathrm{L} / \mathrm{min}$. Small adjustments to the ES voltage were made at each flow rate to keep the ES in the cone-jet mode. The results from detecting the ES current loss and transmission throughout the ESI interface are shown in ${ }^{\circ}$ Figure $4 a$. The ${ }^{\circ}$ experiment ${ }^{\circ}$ was ${ }^{\circ}$ repeated ${ }^{\circ}$ three times and the averaged values along with the standard deviations are plotted. Observation of the ES using a stereo microscope and the relationship of the total ES current to the square root of the flow rate indicated that the cone-jet mode was established for each flow rate [14]..$^{\circ}$ The ${ }^{\circ}$ amount ${ }^{\circ}$ of $^{\circ}$ ion $^{\circ}{ }^{\circ}$ current $^{\circ}{ }^{\circ}$ ost $^{\circ}$ to $^{\circ}$ the ${ }^{\circ}$ front ${ }^{\circ}$ of ${ }^{\circ}$ the interface also steadily increased as the total ES current increased; however, both the transmitted current and the current detected on the inner capillary wall leveled off at around $1.0 \mu \mathrm{L} / \mathrm{min}$, which appears to indicate that a transmission limit was reached for this inlet.

The transmission efficiency of the ESI interface versus $^{\circ}$ flow $^{\circ}$ rate $^{\circ}{ }^{\circ}{ }^{\circ}$ shown ${ }^{\circ}$ in ${ }^{\circ}$ Figure $^{\circ} 4 \mathrm{~b} .^{\circ}{ }^{\circ}$ The ${ }^{\circ}$ transmission efficiency (\% transmission) was calculated from the data ${ }^{\circ}$ in $^{\circ}$ Figure $^{\circ} 4 a^{\circ}$ as $^{\circ}$ the ${ }^{\circ}$ ratio ${ }^{\circ}$ between ${ }^{\circ}$ the ${ }^{\circ}$ transmitted current and the total ES current. The optimal transmis-

a)

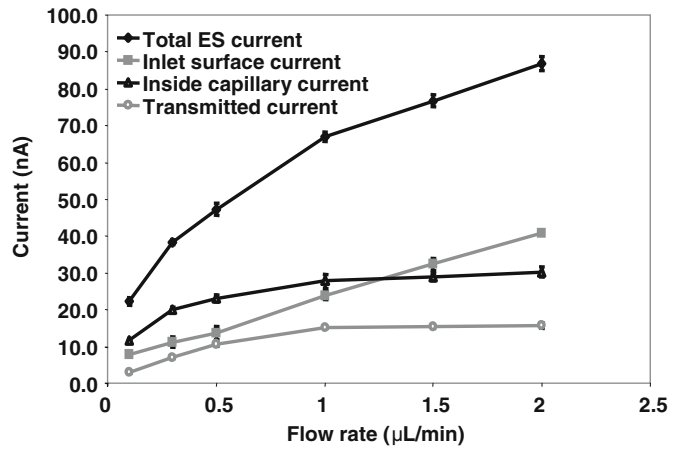

b)

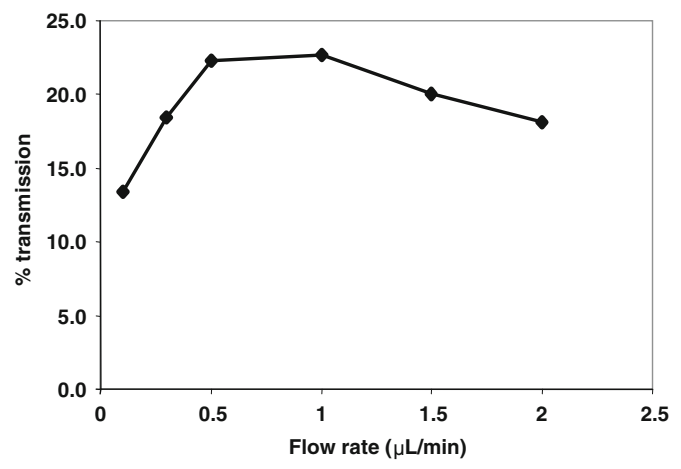

c)

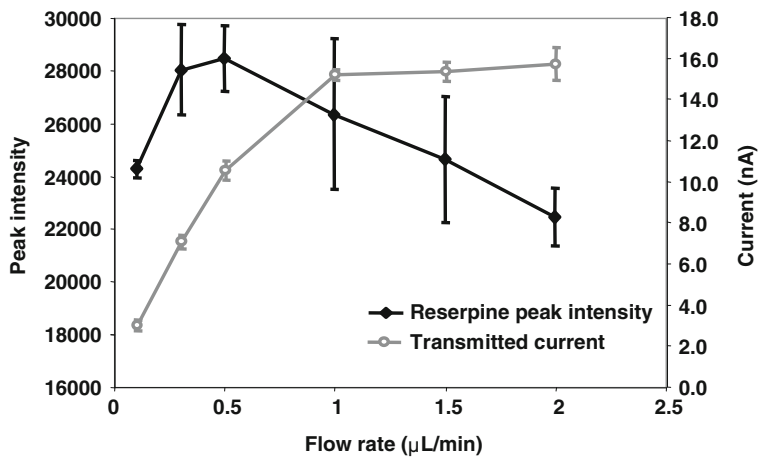

Figure 4. Ionization and transmission efficiency in an ESI interface as a function of the solution flow rate at a 2.0-mm emitter distance to the inlet. (a) Plots of the detected ES current on the front of the interface, the inside wall of the capillary, and transmitted through the capillary. The total ES current is a summation of the current detected on the three charge collectors. (b) The transmission efficiency of the ESI interface as a function of the solution flow rate. The transmission efficiency (\% transmission) was calculated by dividing the transmitted current by the total ES current in a and multiplying by 100. (c) Reserpine peak intensity and transmitted current as a function of solution flow rate. 
sion efficiency of roughly $23 \%$ was achieved at a flow rate of $1.0 \mu \mathrm{L} / \mathrm{min}$. The decreasing efficiency at higher flow rates is mainly attributed to the leveling off of the transmitted current, possibly as the result of an ion transmission limit of the inlet. The decrease in the efficiency at lower flow rates is ascribed, in a large part, to the increased mobilities of the smaller droplets and more gas-phase ions produced by performing ES at lower $^{\circ}$ flow $^{\circ}$ rates $^{\circ}\left[11,{ }^{\circ} 14\right] .{ }^{\circ}$ Because $^{\circ}$ both $^{\circ}$ diffusion $^{\circ}$ and space-charge increase with larger mobilities, loss to the capillary wall increases. This loss can be reduced by decreasing the capillary length (given that the smaller droplets require less heating to desolvate), which will reduce the residence time in the inlet.

Figure $4 c^{\circ}$ plots the reserpine ${ }^{9}$ peak intensity versus 9 flow rate $^{\circ}$ along $^{\circ}$ with $^{\circ}$ the $^{\circ}$ transmitted $^{\circ}$ current $^{\circ}$ from ${ }^{\circ}$ Figure $^{\circ} 4$ a. The peak intensity shows a slight increase as the flow rate is reduced, and it eventually starts to decrease at 0.1 $\mu \mathrm{L} /$ min $^{\circ}{ }^{\circ}$ This $^{\circ}$ effect $^{\circ}$ has $^{\circ}$ been $^{\circ}$ observed $^{\circ}$ previously $^{\circ}[15]$ and is attributed to the large increase in ionization efficiency by operating ES at low flow rates. Schneider et al. $[8]^{\circ}$ observed $^{\circ} \mathrm{a}^{\circ}$ mass-sensitive $^{\circ}$ detector $^{\circ}$ response $^{\circ}$ using reserpine (the peak intensity increased by tenfold when the flow rate was increased from 50 to $500 \mathrm{~nL} / \mathrm{min}$ ), but this required an interface with a very large gas conductance and a high-temperature sheath gas. This work, along with the results presented here, illustrates the importance of solvent desolvation in ionization efficiency and the need for improved ESI interface technologies in this area. We can obtain the losses resulting from incomplete desolvation and ionization by comparing the 1.0 and 0.1 $\mu \mathrm{L} / \mathrm{min}$ flow rates. The reserpine peak intensity is similar at these two flow rates, indicating the amount of gasphase reserpine ions transmitted through the ESI interface to the mass analyzer is similar. However, the amount of ES current transmitted drops from about 15 to 3 nA. From this ratio we can conclude that at the $1.0 \mu \mathrm{L} / \mathrm{min}$ flow rate, at least $80 \%$ of the reserpine transmitted through the inlet does not constitute fully liberated gas-phase ions and therefore does not increase the reserpine peak intensity.

The linear electrode array was used to profile the ES current lost on the front surface of the inlet capillary. Figure ${ }^{\circ}{ }^{\circ}$ plots the ${ }^{\circ}$ current $^{\circ}$ detected $^{\circ}$ on ${ }^{\circ}$ each ${ }^{\circ}$ electrode ${ }^{\circ}$ for the different flow rates used in the previous transmission experiment. Based on these results, the width of the ES plume changes very little as the flow rate and current increase. This indicates that the plume tends to follow the electric field (which changes only slightly for each flow rate because of the small ES voltage adjustments to maintain the cone-jet mode). Conversely, the space-charge effect on the trajectory of the charged droplets/ions in the ES plume seems to be small, unlike the space-charge effect that was observed inside the inlet where there was no electric field. This can be favorable for LC-MS applications where a solution gradient can change the ES current. Because the plume width seems to be more dependent on the electric field and emitter distance, the inlet sampling efficiency should not significantly change at a fixed emitter distance.

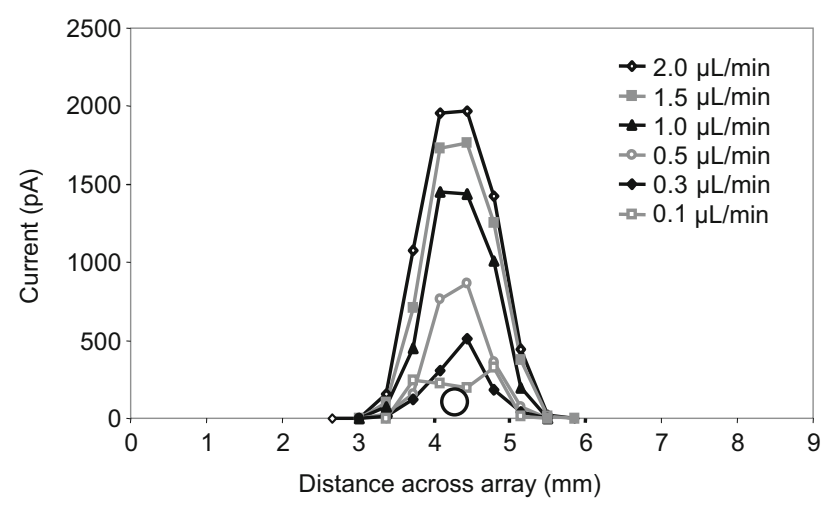

Figure 5. Profiles of the ES current lost to the front of the ESI interface for different solution flow rates. The data points were obtained by detecting the ES current on each electrode in the linear array. The emitter distance to the inlet was $2.0 \mathrm{~mm}$. The drawn circle represents the approximate size and location of the capillary inlet.

\section{Inlet Temperature}

On practically all ESI interfaces, the temperature of the inlet to the mass spectrometer can be optimized for the ES conditions. Typically, higher flow rates require higher temperatures to improve the desolvation of the larger droplets. We investigated the effects of the capillary inlet temperature on the ES current loss and transmission ${ }^{\circ}$ in $^{\circ} \mathrm{an}^{\circ} \mathrm{ESI}^{\circ}$ interface. ${ }^{\circ}$ Figure $^{\circ} 6 \mathrm{a}^{\circ}$ shows $^{\circ}$ the total ES current, the transmitted current, and the current lost to the front surface and inner capillary wall of the interface for the ES of a solvent mixture at a distance of $2 \mathrm{~mm}$ and a flow rate of $0.5 \mu \mathrm{L} / \mathrm{min}$. Both the total $\mathrm{ES}$ current and the current lost to the front surface of the inlet capillary show little change (as expected), but the current hitting the inner wall of the capillary increases and the transmitted current decreases as the temperature ${ }^{\circ}$ increased ${ }^{\circ}$ from $^{\circ} 60^{\circ}$ to $^{\circ} 180^{\circ} \mathrm{C}^{\circ}$ Figure $^{\circ} 6 \mathrm{~b}^{\circ}$ shows ${ }^{\circ}$ the transmission efficiency of the ESI interface as a function of temperature. At low temperatures the transmission efficiency is very high $(\sim 40 \%)$ but drops to about $10 \%$ at $180{ }^{\circ} \mathrm{C}$. This may be a result of the size and number of charged droplets inside the inlet capillary. At low temperatures, the droplets are larger and have lower mobilities, which greatly reduce the loss to the capillary wall. Increasing the temperature improves solvent evaporation, reduces the droplet size, and increases the amount gas-phase ions and clusters. This creates charged species with larger mobilities that more readily collide with the capillary wall.

Using low inlet temperatures to improve the transmission efficiency did not translate into improved MS sensitivity..$^{\circ}$ This $^{\circ}$ is $^{\circ}$ illustrated ${ }^{\circ}$ in $^{\circ}$ Figure $^{\circ} 6 c^{\circ}$ where ${ }^{\circ}$ the reserpine peak intensity is plotted with respect to inlet temperature along with the transmitted current from Figure $^{\circ} 6{ }^{\circ} .^{\circ}$ Below $^{\circ} 150^{\circ} \mathrm{C}^{\circ}$ the ${ }^{\circ}$ peak $^{\circ}$ intensity ${ }^{\circ}$ quickly drops off because of inefficient desolvation. At low temperatures, most of the ES current arises from charged droplets that are not transmitted into the mass analyzer. The reduced peak intensity above $150{ }^{\circ} \mathrm{C}$ can 
a)

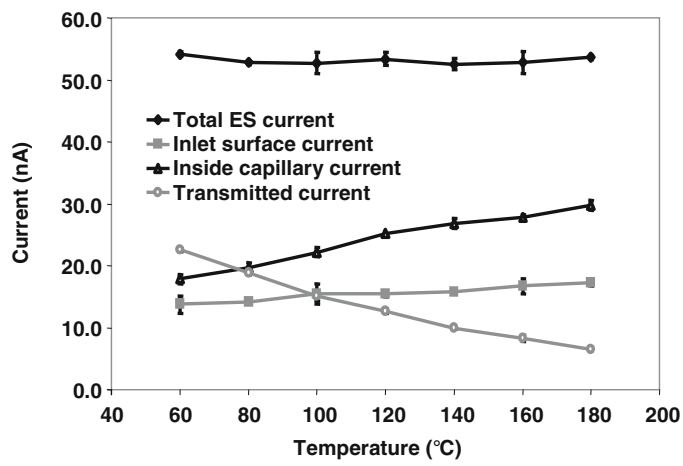

b)

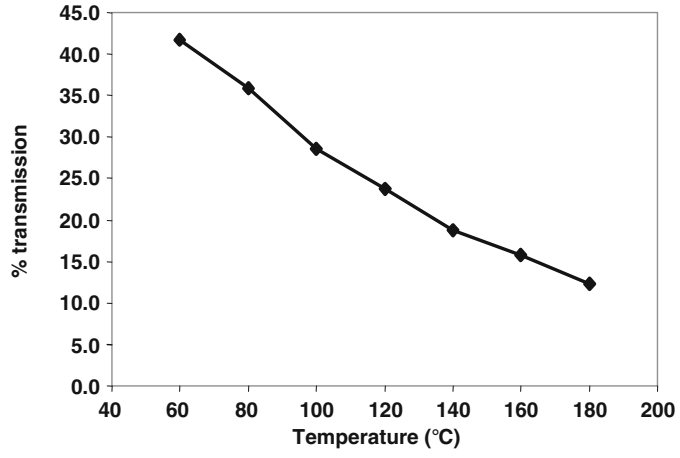

c)

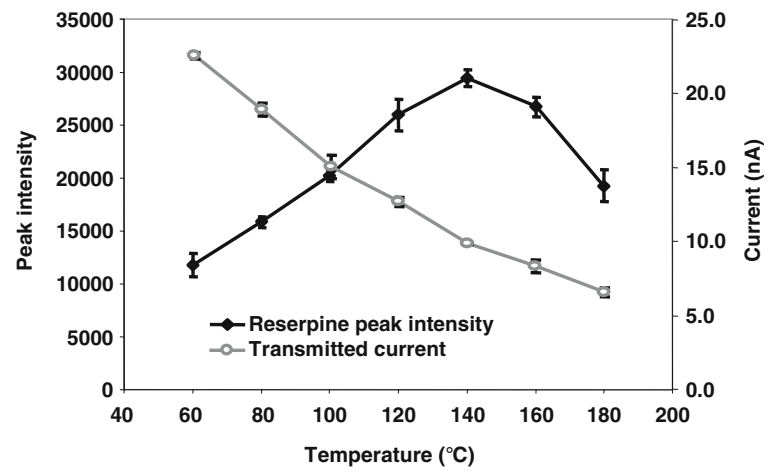

Figure 6. Ionization and transmission efficiency in an ESI interface as a function of inlet temperature. The solution flow rate was $0.5 \mu \mathrm{L} / \mathrm{min}$ and the emitter distance to the inlet was $2.0 \mathrm{~mm}$. (a) Plots of the detected ES current on the front of the interface, the inside wall of the capillary, and transmitted through the capillary. The total ES current is a summation of the current detected on the three charge collectors. (b) The transmission efficiency of the ESI interface as a function of the inlet temperature. The transmission efficiency (\% transmission) was calculated by dividing the transmitted current by the total ES current in a and multiplying by 100 . (c) Reserpine peak intensity and transmitted current as a function of inlet temperature.

be caused by several factors. First, diffusion increases as the temperature increases. Second, the gas throughput (i.e., mass flow rate) in the inlet capillary decreases as the temperature increases, as the result of a lower density of the background gas. Third, more gas-phase ions are created closer to the capillary entrance, which increases the residence time of the higher mobility species and increases the ion loss to the wall. The true cause for the decreased peak intensity at higher temperature in this experiment is likely a combination of all three factors.

\section{Conclusion}

To reduce ion losses in an ESI-MS and improve instrument sensitivity, it is important to better understand and characterize ionization and transmission efficiency properties of the ESI interface. We have accomplished this using a combination of three approaches. First, we spatially profiled the ES current lost to the front of the interface. Second, we detected the ES current loss on the front of the instrument, the loss inside the heated inlet capillary, and the amount of current transmitted into the first vacuum region of the mass spectrometer. Third, we used the peak intensity of a model analyte to determine the efficiency of ion production.

By performing a series of experiments that varied ES conditions, a clearer picture of the ES process and the ES current transmission in a common ESI interface has emerged. An important result was the advantage of using lower ES flow rates. The production of smaller droplets allows the emitter to be placed closer to the instrument where we observed that $>90 \%$ of the ES current can be effectively sampled into the inlet. The decreased ES current from low flow rate electrosprays reduces space-charge effects in the ion source and increases the transmission efficiency of a capillary inlet. We also found that analyte peak intensity can increase as the flow rate is decreased. Increasing the flow rate to increase the amount of analyte transmitted into the interface did not produce a larger peak intensity in our experiments. The additional analyte was lost to incomplete ionization $(>80 \%$ of reserpine transmitted through the inlet using a flow rate of $1.0 \mu \mathrm{L} / \mathrm{min}$ was not detected at the $\mathrm{M}+\mathrm{H}$ peak).

At higher flow rates, a longer ES emitter-to-inlet distance is necessary to allow for charged droplet desolvation and for more droplet fission events. We found that the width of the ES plume quickly expands to $>7 \mathrm{~mm}$ at a distance of $5 \mathrm{~mm}$ to the inlet when electrospraying a solution at $0.5 \mu \mathrm{L} / \mathrm{min}$. The large discrepancy between the size of the plume and the area sampled by the inlet can lead to large losses. To reduce this loss, a multi-inlet can be used to increase the sampling ${ }^{\circ}$ area [39], ${ }^{\circ}$ and ${ }^{\circ}{ }^{9}$ multi-ESI ${ }^{\circ}$ emitter ${ }^{\circ}$ array ${ }^{\circ}$ can be used to obtain the benefits of nano-ESI at higher solution ${ }^{\circ}$ flow ${ }^{\circ}$ rates $[40]$. The ${ }^{\circ}$ temperature ${ }^{\circ}$ of $^{\circ}$ the inlet ${ }^{\circ}$ should also be optimized for the higher flow rate. We observed that increasing the temperature decreased the amount of ES current transmitted through the ESI interface; however, analyte peak intensity increased as more gas-phase ions were produced. The optimum temperature also depends on the stability of the analyte, given that disassociation can occur (e.g., phosphopeptides and noncovalent complexes are more labile).

This work illustrates some of the complexities in ESI for MS analyses. The best sensitivities can be achieved only when both the ionization and transmission efficiencies are optimized, and improvements in one can adversely affect the other. For example, moving the ES emitter closer to the inlet to improve ES current sam- 
pling can lead to decreased solvent evaporation and ion production. In addition, it is easier to transmit charged droplets in the area of the interface dominated by gas dynamic transport, and it is easier to transmit ions in low-pressure regions that use radio-frequency ion guides. Therefore, the location at which the analyte ions are created (before the inlet, inside the inlet, or after the inlet) can lead to different loss rates. Many of these results can be applied to other interface geometries, such as an inlet orifice with a curtain gas. Here, the sampling inefficiency resulting from the expanding ES plume is similar to the capillary inlet; however, desolvation before the inlet plays a greater role in ion production arising from the cooling effects of the gas jet expansion directly behind the thin orifice. Overall, this work provides a foundation for improving ESI interfaces and directing the optimization of ES conditions to improve ionization and transmission efficiencies.

\section{Acknowledgments}

The authors thank Dr. Ioan Marginean for helpful discussions and editing of the manuscript. Portions of this research were supported by the NIH National Center for Research Resources (RR-018522), the NIH National Cancer Institute (R21 CA-126191), and the National Institute of Allergy and Infectious Diseases $\mathrm{NIH} / \mathrm{DHHS}$ through interagency agreement Y1-AI-4894-01. Experimental portions of this research were performed in the Environmental Molecular Sciences Laboratory, a U.S. DOE national scientific user facility located at the Pacific Northwest National Laboratory (PNNL) in Richland, Washington. PNNL is a multiprogram national laboratory operated by Battelle for the DOE under Contract No. DE-AC05-76RLO 1830.

\section{References}

1. Yamashita, M.; Fenn, J. B. Electrospray Ion Source. Another Variation on the Free-Jet Theme. J. Phys. Chem. 1984, 88, 4451-4459.

2. Fenn, J. B.; Mann, M.; Meng, C. K.; Wong, S. F.; Whitehouse, C. M. Electrospray Ionization-Principles and Practice. Mass Spectrom. Rev. 1990, 9, 37-70.

3. Qian, W. J.; Jacobs, J. M.; Liu, T.; Camp, D. G.; Smith, R. D. Advances and Challenges in Liquid Chromatography-Mass Spectrometry-Based Proteomics Profiling for Clinical Applications. Mol. Cell Proteomics 2006, 5, 1727-1744.

4. Shen, Y.; Zhang, R.; Moore, R. J.; Kim, J. K.; Metz, T. O.; Hixson, K. K.; Zhao, R.; Livesay, E. A.; Udseth, H. R.; Smith, R. D. Automated 20 Kpsi RPLC-MS and MS/MS with Chromatographic Peak Capacities of 1,000-1,500 for and Capabilities for Proteomics and Metabolomics. Anal. Chem. 2005, 77, 3090-3100.

5. Swanson, S. K.; Washburn, M. P. The Continuing Evolution of Shotgun Proteomics. Drug Discov. Today. 2005, 10, 719-725.

6. Shen, Y.; Smith, R. D. Advanced Nanoscale Separations and Mass Spectrometry for Sensitive High-Throughput Proteomics. Exp. Rev. Proteomics 2005, 2, 431-447.

7. Kebarle, P.; Tang, L. From Ions in Solution to Ions in the Gas PhaseThe Mechanism of Electrospray Mass Spectrometry. Anal. Chem. 1993, 65, A972-A986

8. Schneider, B. B.; Javaheri, H.; Covey, T. R. Ion Sampling Effects under Conditions of Total Solvent Consumption. Rapid Commun. Mass Spectrom. 2006, 20, 1538-1544.

9. Cole, R. B. Some Tenets Pertaining to Electrospray Ionization Mass Spectrometry. J. Mass Spectrom. 2000, 35, 763-772.

10. Cech, N. B.; Enke, C. G. Practical Implications of Some Recent Studies in Electrospray Ionization Fundamentals. Mass Spectrom. Rev. 2001, 20, 362-387.

11. Wilm, M. S.; Mann, M. Electrospray and Taylor-Cone Theory, Dole's Beam of Macromolecules at Last? Int. J. Mass Spectrom. Ion Processes 1994, 136, 167-180.

12. Wahl, J. H.; Goodlett, D. R.; Udseth, H. R.; Smith, R. D. Use of Small-Diameter Capillaries for Increasing Peptide and Protein Detection Sensitivity in Capillary Electrophoresis-Mass Spectrometry. Electrophoresis $1993,14,448-457$.
13. Goodlett, D. R.; Wahl, J. H.; Udseth, H. R.; Smith, R. D. Reduced Elution Speed Detection for Capillary Electrophoresis Mass-Spectrometry. J. Microcolumn. Sep. 1993, 5, 57-62.

14. Fernandez de la Mora, J.; Loscertales, I. The Current Emitted by Highly Conducting Taylor Cones. J. Fluid Mech. 1994, 260, 155-184.

15. Tang, K.; Page, J. S.; Smith, R. D. Charge Competition and the Linear Dynamic Range of Detection in Electrospray Ionization Mass Spectrometry. J. Am. Soc. Mass Spectrom. 2004, 15, 1416-1423.

16. Schmidt, A.; Karas, M.; Dulcks, T. Effect of Different Solution Flow Rates on Analyte Ion Signals in Nano-ESI MS, or: When Does ESI Turn into Nano-ESI? J. Am. Soc. Mass Spectrom. 2003, 14, 492-500.

17. Cole, R. B., Ed. Electrospray Ionization Mass Spectrometry: Fundamentals, Instrumentation, and Applications. John Wiley \& Sons: New York, 1997.

18. Chowdhury, S. K.; Katta, V.; Chait, B. T. An Electrospray-Ionization Mass Spectrometer with New Features. Rapid Commun. Mass Spectrom. 1990, 4, 81-87.

19. Li, L. Y. T.; Campbell, D. A.; Bennett, P. K.; Henion, J. Acceptance Criteria for Ultratrace HPLC-Tandem Mass Spectrometry: Quantitative and Qualitative Determination of Sulfonylurea Herbicides in Soil. Anal. Chem. 1996, 68, 3397-3404.

20. Allanson, J. P.; Biddlecombe, R. A.; Jones, A. E.; Pleasance, S. The Use of Automated Solid Phase Extraction in the "96 Well" Format for High Throughput Bioanalysis Using Lipid Chromatography Coupled to Tandem Mass Spectrometry. Rapid Commun. Mass Spectrom. 1996, 10, $811-816$.

21. Wilm, M.; Mann, M. Analytical Properties of the Nanoelectrospray Ion Source. Anal. Chem. 1996, 68, 1-8.

22. Smith, R. D.; Loo, J. A.; Edmonds, C. G.; Barinaga, C. J.; Udseth, H. R. New Developments in Biochemical Mass Spectrometry: Electrospray Ionization. Anal. Chem. 1990, 62, 882-899.

23. Kim, T.; Udseth, H. R.; Smith, R. D. Improved Ion Transmission from Atmospheric Pressure to High Vacuum Using a Multi-Capillary Inlet and Electrodynamic Ion Funnel Interface. Anal. Chem. 2000, 72, 50145019.

24. Bruins, A. Mass Spectrometry with Ion Sources Operating at Atmospheric Pressure. Mass Spectrom. Rev. 1991, 10, 53-77.

25. Kim, T.; Tolmachev, A. V.; Harkewicz, R.; Prior, D. C.; Anderson, G. A.; Udseth, H. R.; Smith, R. D.; Bailey, T. H.; Rakov, S.; Futrell, J. H. Design and Implementation of a New Electrodynamic Ion Funnel. Anal. Chem. 2000, 72, 2247-2255.

26. Shaffer, S. A.; Tang, K.; Anderson, G. A.; Prior, D. C.; Udseth, H. R.; Smith, R. D. A Novel Ion Funnel for Focusing Ions at Elevated Pressure Using Electrospray Ionization Mass Spectrometry. Rapid Commun. Mass Spectrom. 1997, 11, 1813-1817.

27. Page, J. S.; Tolmachev, A. V.; Tang, K.; Smith, R. D. Theoretical and Experimental Evaluation of the Low $\mathrm{m} / \mathrm{z}$ Transmission of an Electrodynamic Ion Funnel. I. Am. Soc. Mass Spectrom. 2006, 17, 586-592.

28. Julian, R. R.; Mabbett, S. R.; Jarrold, M. F. Ion Funnels for the Masses: Experiments and Simulations with a Simplified Ion Funnel. J. Am. Soc. Mass Spectrom. 2005, 16, 1708-1712.

29. Lynn, E. C.; Chung, M. C.; Han, C. C. Characterizing the Transmission Properties of an Ion Funnel. Rapid Commun. Mass Spectrom. 2000, 14, 2129-2134

30. Gerlich, D. State-Selected and State-to-State Ion-Molecule Reaction Dynamics. Part 1. Experiment. Wiley: New York, 1992; pp 1-76.

31. Kebarle, P. A Brief Overview of the Present Status of the Mechanisms Involved in Electrospray Mass Spectrometry. J. Mass Spectrom. 2000, 35, $804-817$.

32. Bruins, A. P. Mechanistic Aspects of Electrospray Ionization [Review]. J. Chromatogr. 1998, 794, 345-357.

33. Thompson, J. W.; Eschelbach, J. W.; Wilburn, R. T.; Jorgenson, J. W. Investigation of Electrospray Ionization and Electrostatic Focusing Devices Using a Three-Dimensional Electrospray Current Density Profiler. J. Am. Soc. Mass Spectrom. 2005, 16, 312-323.

34. Busman, M.; Sunner, J.; Vogel, C. R. Space-Charge-Dominated MassSpectrometry Ion Sources-Modeling and Sensitivity. J. Am. Soc. Mass Spectrom. 1991, 2, 1-10.

35. Manisali, I.; Chen, D. D. Y.; Schneider, B. B. Electrospray Ionization Source Geometry for Mass Spectrometry: Past, Present, and Future. Trends Anal. Chem. 2006, 25, 243-256.

36. Lin, B. W.; Sunner, J. Ion Transport by Viscous Gas Flow through Capillaries. J. Am. Soc. Mass Spectrom. 1994, 5, 873-885.

37. Kelly, R. T.; Page, J. S.; Luo, Q.; Moore, R. J.; Orton, D. J.; Tang, K.; Smith R. D. Chemically Etched Open Tubular and Monolithic Emitters for Nanoelectrospray Ionization Mass Spectrometry. Anal. Chem. 2006, 78, 7796-7801.

38. Shaffer, S. A.; Tolmachev, A.; Prior, D. C.; Anderson, G. A.; Udseth, H. R.; Smith, R. D. Characterization of a New Electrodynamic Ion Funnel Interface for Electrospray Ionization Mass Spectrometry. Anal. Chem. 1999, 71, 2957-2964.

39. Kim, T.; Tang, K.; Udseth, H. R.; Smith, R. D. A Multi-Capillary Inlet Jet Disruption Electrodynamic Ion Funnel Interface for Improved Sensitivity Using Atmospheric Pressure Ion Sources. Anal. Chem. 2001, 73, 4162-4170.

40. Kelly, R. T.; Page, J. S.; Tang, K.; Smith, R. D. Array of Chemically Etched Fused Silica Emitters for Improving the Sensitivity and Quantitation of Electrospray Ionization Mass Spectrometry. Anal. Chem. 2007, $79,4192-4198$. 